\title{
GPPS-CH-2020-0071
}

\section{APPLICATION OF A GLOBAL SENSITIVITY SCREENING METHOD FOR DESIGN SPACE EXPLORATION OF COUPLED TURBOFAN ENGINE PERFORMANCE AND PRELIMINARY DESIGN}

\author{
Jens Schmeink, Alexander Görtz, Jannik Häßy \\ German Aerospace Center (DLR) \\ Institute of Propulsion Technology \\ jens.schmeink@dlr.de \\ Cologne, Germany
}

\begin{abstract}
Multidisciplinary preliminary engine design involves a large number of tools and participants. The highly iterative and multidisciplinary workflows make it difficult to evaluate the influence of individual design parameters on the final result.

With high-dimensional parameter studies the design space of the system can be investigated. However, the evaluation of the results can rapidly become very challenging. The screening method of elementary effects according to Morris as a method of global sensitivity analysis (GSA) can provide useful insights from even a small number of evaluations. Especially a reduction of the dimensionality due to factor fixing of input parameters with low influence on the problem and factor prioritization of important parameters are addressed by GSA. The purpose of the paper is to demonstrate the application of the method by means of a coupled process which includes performance calculations of the entire engine system and preliminary design of a high-pressure compressor. The variation of input parameters of the different disciplines will be investigated and it will be shown that the Morris screening characteristics allow the evaluation of parameters within the design space in particular with regard to a classification of the significance for the result variables. This shows in the application example that in the comparison parameters with a clear influence emerge from both disciplines.
\end{abstract}

\section{INTRODUCTION}

Increasingly large process chains make it more and more difficult to identify correlations between various design parameters of different disciplines in multidisciplinary preliminary design. At the same time, it is very valuable for the multidisciplinary view of systems to know to what extent which parameters from which discipline have influence on output parameters. Such findings can be incorporated into optimization processes. There, parameters with little influence can be kept constant to reduce the number of expensive system evaluations. Therefore it is not surprising that particularly in the context of the Multidisciplinary optimization (MDO) different authors have dealt with procedures to evaluate the relations of input and output variables. Chiba et al. (2005) used a Self-Organizing-Map (SOM) and an Analysis of Variance (ANOVA) technique to analyse the influence of input parameters for a MDO of a regional-jet wing. The advantages and disadvantages of these methods are discussed and illustrated there. The SOM approach cannot show interactions of the design parameters and the ANOVA gives an indicator which input parameter influences the output parameters but not "how". Khurana and Winarto (2010) use Morris screening as a pre process to reduce number of input parameters for an aerofoil shape design optimization.

Arsenyev et al. (2014) used Morris screening and Sobol indices as another GSA method in the context of vane cluster optimization. There, the advantages of these methods are emphasized over those that only cover linear relationships, since otherwise corresponding misjudgements of the significance of parameters could be made. In this paper, the focus will be on how straightforward GSA procedures can provide a valuable overview of the discipline-specific input parameters and relevant output variables. Thereby the focus is explicitly on the preliminary design. Studies in this field have been carried out in different disciplines. For example, in the field of civil engineering GSA methods for the analysis of the parameter space were shown in Østergård et al. (2017). In the same area Nembrini et al. (2014) explicitly used Morris screening for such analyses. In the field of turbo machinery Antinori 
et al. (2013) used different GSA methods including Morris screening supported by Polynomial Chaos Expansion for the evaluation of the secondary air system of a jet engine low pressure turbine. Morris screening and typically more expensive Sobol' Indices showed results in good agreement in that study. All these publications thus show that the GSA, especially the Morris Screening, can be a valuable extension of interpretation processes. However, the applicability has not yet been demonstrated and evaluated for the coupled preliminary engine design.

In this paper the application of a fully coupled thermodynamic design of the engine with aerodynamic 1-D design of the high pressure compressor (HPC) is investigated by means of Morris screening. The coupling is achieved by an iterative feedback of the compressor design and its off-design behaviour via a characteristic map to the performance model.

The description of the applied process will be shown in the methodology section after a short description of the Morris screening method. This will be followed by a presentation of exemplary results and a discussion of the approach.

\section{METHODOLOGY}

In the following, the various elements of the demonstrated process will be described. Basics about Morris screening are explained first, then the thermodynamic cycle and aerodynamic compressor design methodology is presented and finally the coupling is described.

\section{Background of Morris screening}

GSA is often associated with uncertainty quantification. The goal of GSA is mostly to correlate distributions in result variables with the distributions of input parameters and thus to assess their significance. In this way, global (i.e. in the entire parameter space spanned by all input variables) relationships can be identified and used for the interpretation of the underlying system. This is important in particular to neglect the variation of individual parameters in the future, if these show no or only small influence. There are different methods which are assigned to the topic of GSA. For a more comprehensive and detailed overview, reference is made to Saltelli et al. (2008) and Iooss and Lemaître (2015).

A method that requires comparatively few evaluations of the process to be analysed is the method of Elementary Effects (EEs) according to Morris (1991) and improved by Campolongo et al. (2005). It is based on an efficient way of screening the parameter space and determining meaningful parameters from the results of individual evaluations of the model.

For the sampling of the parameter space with $k$ input parameters and thus $k$ dimensions the design space is considered as a uniform grid with $p$ levels. The grid is now screened by so-called trajectories. Each of these consists of a random starting point in the grid and then following $k$ steps in the grid to vary each parameter once in random order. This is shown in a simplified form for three trajectories and three input parameters in Fig. 1 . In each case, one step is made by the difference $\Delta$. This $\Delta$ is defined by eq. 1 in order to scan the space as evenly as possible.

$$
\Delta=p /[2(p-1)]
$$

To take into account the range of values of the individual parameters, the grid is converted for each input parameter examined, whereby distribution functions can also be applied, for example normal distributions. In this case, the values for $\Delta$ associated with this parameter must also be adjusted. The possible consideration of specific distributions is another advantage of this method compared to local sensitivities or a classical experimental design.

Two evaluations along the trajectory always represent the change of a parameter. The definition of so called EE for a parameter $i$ is defined in eq. 2 as the difference of two evaluations of the points $l$ and $l+1$ divided by the step size $\Delta$ for an step with an increasing $\Delta$.

$$
E E_{i}^{j}\left(x^{(l)}\right)=\frac{\left[y\left(x^{(l+1)}-y\left(x^{(l)}\right)\right]\right.}{\Delta} .
$$

The EEs of all $r$ trajectories are collected as results in three key indicators as defined in the equations 3 to 5 .

$$
\begin{aligned}
& \mu_{i}=\frac{1}{r} \sum_{j=1}^{r} E E_{i}^{j} . \\
& \mu_{i}^{*}=\frac{1}{r} \sum_{j=1}^{r}\left|E E_{i}^{j}\right| .
\end{aligned}
$$




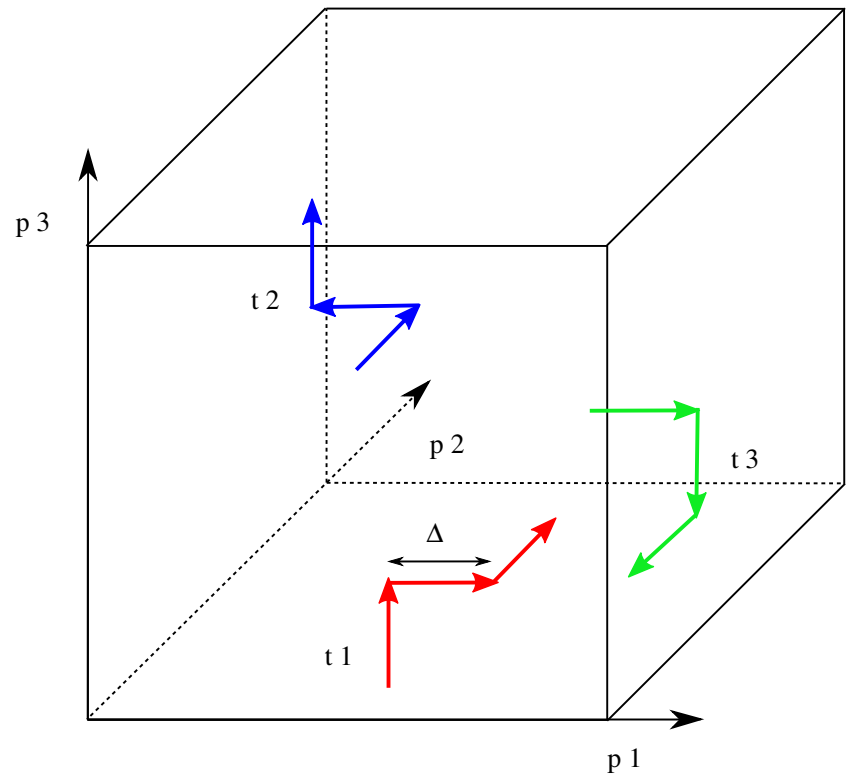

Figure 1: Exemplary illustration of the sampling plan for three dimensions of parameters $\mathrm{p} 1$ to $\mathrm{p} 3$ with three trajectories t1 to $\mathrm{t} 3$

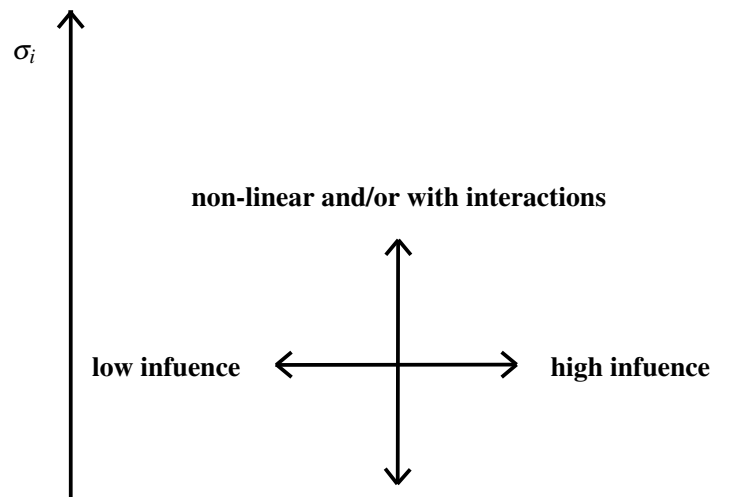

linear and without interactions

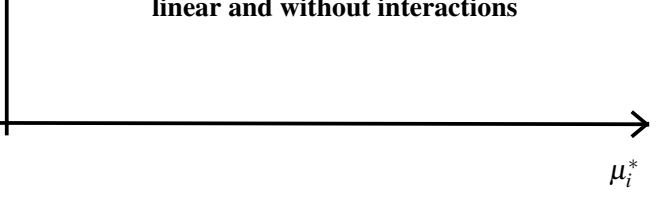

Figure 2: Scheme of evaluation diagram for Morris screening

$$
\sigma_{i}^{2}=\frac{1}{r-1} \sum_{j=1}^{r}\left(E E_{i}^{j}-\mu_{i}\right)^{2}
$$

$\mu_{i}^{*}$ is often referred as a general measure of importance of the parameter. It is numerical defined as the mean value of the absolute values of the EEs of the parameter $i$ while $\mu_{i}$ is the mean value of the EEs themselves. Identical values for $\mu_{i}^{*}$ and the absolute value of $\mu_{i}$ indicates a monotone behaviour of the model. $\sigma_{i}$ corresponds to the standard deviation of the EEs and is an indicator for non-linear behaviour or interaction between the input parameters.

The usual method to process the characteristic values graphically is to display the values for $\sigma_{i}$ over $\mu_{i}^{*}$ for all input parameters as presented in Fig. 2. With this representation, the influence of the different input variables relative to each other can be easily estimated. In particular, the range of small values for $\mu^{*}$ and $\sigma$ in the figure shows which parameters are of secondary importance for the characteristic of the output variable.

\section{COUPLED MODEL SIMULATION}

The central element of the analysis is the underlying model to be investigated. The model examined here represents a coupling of a thermodynamic analysis of the engine's overall system and a mean-line simulation of the high-pressure compressor.

Such a coupling allows insights into certain relationships that the individual tools do not provide. For example, Kim et al. (2015), who also coupled a mean-line tool with a performance tool, showed that the settings for variable guide vanes (VGVs) can only be set meaningfully related to engine overall performance. Using a 2-D through flow tool, Reitenbach et al. (2015) had also confirmed this.

\section{Performance Model}

The engine performance calculation is done by DLR's in-house tool DLR Performance Program (DLRp2). This tool uses a modular component-based structure to model different engine concepts. Components, e.g. compressors, turbines and combustor, are interconnected to build a whole engine and the operational behaviour can be evaluated for both the design and off-design case. For the off-design case generic component maps are applied. A more detailed overview is given by Becker et al. (2011).

In this study a performance model for a two-spool unmixed turbofan engine is used. The parameter variations that are performed within the GSA are starting from the thermodynamic cycle of a reference engine. As reference serves a design that is inspired by the PW1100G engine.

A set of design laws for the thermodynamic cycle calculation is applied to ensure feasible engine designs when parameter variations are made. This set takes into account major physical dependencies between the engine components and will be described in the following. 
For each thermodynamic engine design the ideal fan pressure ratio (FPR) is used. The ideal FPR is derived by the condition for unmixed turbofan engines that the ratio of bypass nozzle to core nozzle velocity equals the engine transfer efficiency. A decreasing FPR towards the hub is assumed by keeping a constant ratio between the core and the bypass section.

The polytropic efficiency of turbo components are modelled by statistical approaches according to Grieb (2004). The efficiency of the fan depends on the selected FPR. Starting from the reference values, the efficiencies of the other turbo components are corrected for the component size, which is given by the reduced mass flow.

The cooling air demand for the high pressure turbine (HPT) is estimated for the maximum take-off (MTO) condition. Therefore the stator outlet temperature of the HPT as well as the temperature of the cooling air are taken into account as described by Grieb (2004). The ratio of working to non-working cooling air mass flow is kept constant.

The rotational speed of the high pressure shaft is determined by keeping a constant area-averaged circumferential velocity $\left(\bar{u}_{c, I n}\right)$ for the first rotor of the HPC. For the speed of the low pressure shaft the reduced circumferential velocity at the fan tip is modelled in dependence on the FPR. (Grieb (2004)). The respective radius is calculated by means of a prescribed hub-to-tip-ratio and a Mach number with which the flow area is estimated.

The overall pressure ratio is reached by adjusting the pressure ratios of the booster and the HPC. The pressure split of the reference engine is maintained. It is defined as the pressure ratio of the HPC over the sum of the pressure ratios of HPC and low pressure compressor (LPC). The outlet temperature of the combustor is set by the fuel-to-air-ratio.

\section{Mean-line Simulation}

The one-dimensional mean-line simulation tool is implemented as a process element in the gas turbine design framework GTlab Reitenbach et al. (2020). In the following the focus is on special features of the applied mean-line calculation and a brief description of the input and output parameters used in the GSA.

Important input parameters of the mean-line calculation are various geometric descriptions of the annulus and the 2-D blade geometry via data curves and splines, an initial assumption for the number of stages, the thermodynamic inlet conditions, the rotational speed, the target pressure ratio and various parameters for the description of the aerodynamic design (deHaller number, diffusion number, stator outlet angles) including the specification of the load of the individual stages.

For this purpose, a distribution of the maximum load coefficient is specified. It is defined according to eq. 6 with the ratio of the total enthalpy change $\Delta h_{t}$ and the rotational speed $u$.

$$
\psi_{h}=\frac{\Delta h_{t}}{\frac{u^{2}}{2}}
$$

If the design shows that the currently assumed distribution of $\psi_{h}$ is not sufficient to achieve the predefined pressure ratio, an additional stage is considered in the design. This is inserted in the second last position in order not to distort the possible special definitions of the specifications of the last stage like axial outflow.

The implemented loss models are based on different references. The profile loss models is founded on Lieblein et al. (1953), side wall loss model on Eftari et al. (2011). The secondary loss model is based on Templalexis et al. (2011) and a model of Banjac et al. (2015) was used for tip clearance loss.

In order to represent the geometry of the compressor more realistically and to build upon the experience of existing designs, a parametric approach has been selected for the geometrical specifications of the design process. The mean-line path is modelled as a B-Spline within the process. Likewise, various quantities for the parametrization of the 2D geometry, such as the aspect ratio of the blade rows are described via data curves. All data for the curves can be extracted from existing designs. In addition, a B-spline is first assumed to describe the annulus height in the compressor and then iteratively updated by the results of the aerodynamic design. The splines or data curves are normalized to boundary conditions as required. In this way, a matching between the aerodynamic results for the flow annulus and the geometry is iteratively achieved in the design process.

For the application in the GSA process a high pressure compressor that has been designed with the through flow code ACDC (Schnoes and Nicke (2015)) is selected. The recalculation of the compressor using the mean-line tool shows good agreement with the reference data.

To improve the operational behaviour of the compressor at part load, adjustments of the variable stators can be mapped via stored schedules. Depending on the reduced relative speed of the compressor, the vanes are changed accordingly. During the design process the reference incidences of the blades are parameters to manipulate off-design behaviour as well. While a value of zero leads to minimal losses in design point a deviation can have a positive influence on the off-design efficiency or surge margin (SM).

The use of a mean-line method was chosen here because it allows a full coupling and at the same time requires short computing times. The informative value with regard to efficiencies or the surge margin is limited, but only trends that are mapped by the meanline code are included in the evaluation of the morris screening via the EEs. 


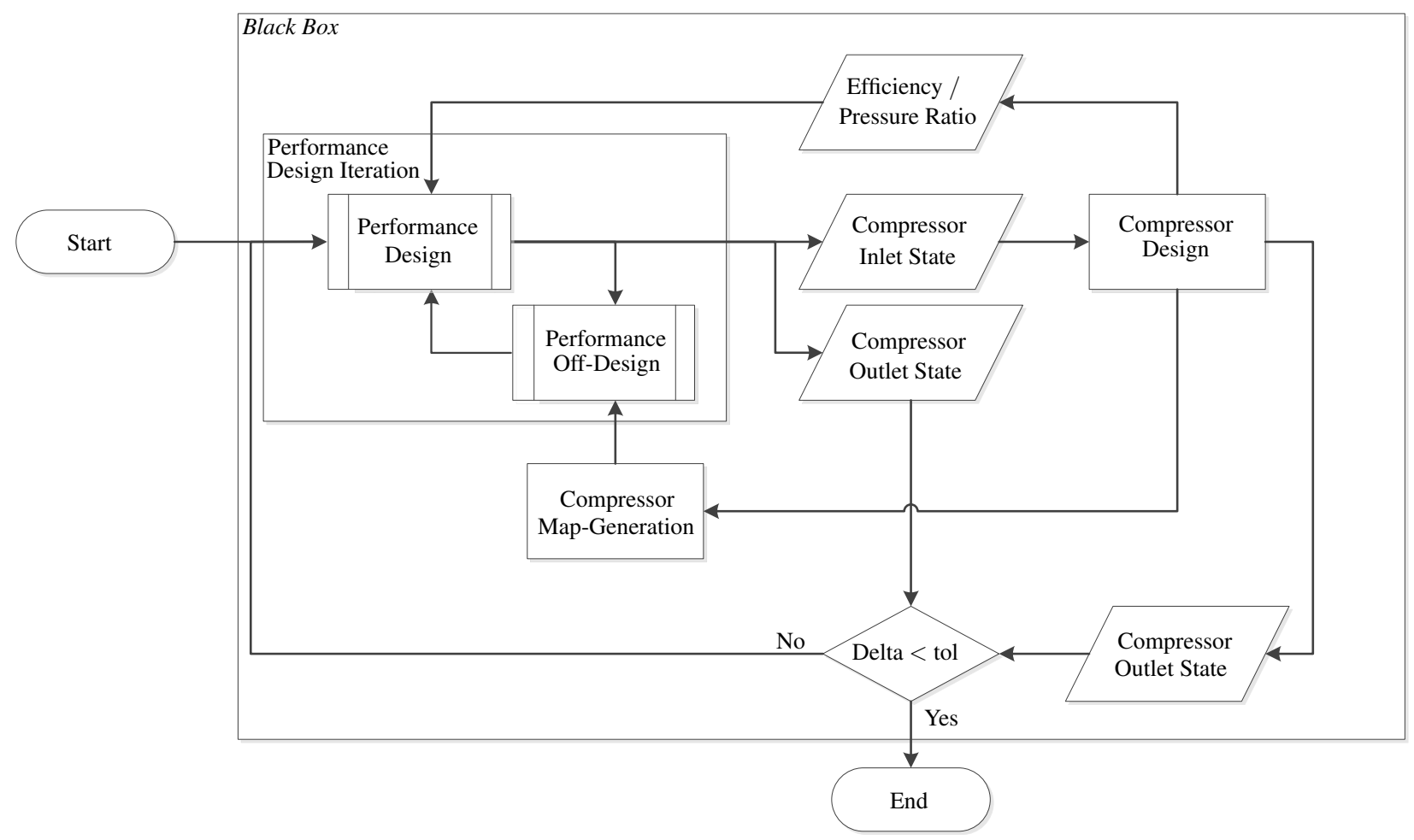

Figure 3: Scheme of the coupling of performance and aerodynamic design simulations

\section{Overall Process}

The coupling of the performance calculation and the mean-line process is illustrated in Fig. 3. This represents the Black Box, which is the system to be analysed by the Morris screening.

The overall simulation process is iterative. It consists of the performance calculation with simulation of design and off-design operating points as described in the previous section. Thermodynamic states at the inlet and outlet of the compressor are collected. The inlet state is used as boundary conditions for the mean-line tool to calculate the compressor design. The isentropic efficiency of the mean-line results is returned to the performance model as well as a compressor map generated based on the current aerodynamic design. This is used to calculate the off-design performance points. The iteration between thermodynamic and aerodynamic design is performed until a match within a given accuracy of the outlet state is achieved. At least two iterations are evaluated to ensure full coupling. Afterwards the model can offer result variables of the different disciplines for the evaluation of the applied input variables:

Thermodynamic values like the thrust specific fuel consumption (TSFC) at design and off-design operating points can give an overview of the overall engine performance. The SM of the HPC based on the maps applied in the thermodynamic models are an indicator for compressor stability. The number of stages of the compressor needed by the mean-line tool to fulfil the requirements can be an indicator for weight estimations of the compressor. The tools used are not sufficient to give a well-founded value for the SM, but can provide a rough estimate. An exact determination is not part of this work. However, especially with this output variable very different input variables play a role, so that the interaction results in a suitable observation case to demonstrate the screening procedure.

The SM is defined in this work according to equation 7.

$$
S M=\frac{\Pi_{S L}-\Pi_{O P}}{\Pi_{O P}-1} * 100 \%
$$

The pressure ratios at the considered operating point are set in relation to the pressure ratio at the surge line at the same reduced mass flow.

For each point of the sample, based on the trajectories, the Black Box is evaluated. The results of each individual system execution are then collected to determine the Morris screening characteristics in conjunction with the underlying sample. 


\section{RESULTS AND DISCUSSION}

The approach of a complete coupling via the component map applied here can be solved iteratively for all boundary conditions applied in the course of this work. Between 2 and 4 iterations are necessary. The time required for the mean-line compressor design with the map calculation and the performance iteration are roughly equal.

Of key importance of any global sensitivity analysis is always the choice of the distributions or value ranges of the input parameters. Therefore it should be emphasized that the following results are only valid for exactly the coupled processes described here and the boundary conditions such as parameter distributions of this study. In addition, the experimental designs used in Morris screening are based on randomly selected starting points of the trajectories and random sequences of the changed parameters. In the small sample that accompanies the procedure, differences in the evaluations based on these randomness may be present.

Firstly, the conclusions of the Morris screening for the system under investigation will be discussed. Afterwards, assessments regarding the method itself are presented. The analyses are carried out using the methods presented above, taking into account 34 input parameters with predefined value ranges.

On the one hand, these are parameters from the design of the engine performance for the design point respectively MTO as shown in table 2. On the other hand, parameters from the design process of the mean-line tool are examined as variable input parameters, see table 3 . The value ranges were determined on the basis of experience as small deviations around the basic design of the engine respectively the compressor. The mentioned operating points are defined as given in table 1.

Table 1: Parameter variation of the performance model. Boundary conditions of the design point and MTO as mentioned exemplary operating point

\begin{tabular}{lccc}
\hline Parameter & Design & MTO & Unit \\
\hline Thrust & 22.8 & 114.3 & {$[\mathrm{kN}]$} \\
\hline Fligth level & 10668 & 0 & {$[\mathrm{~m}]$} \\
\hline Delta to ISA temperature & 10 & 10 & {$[\mathrm{~K}]$} \\
\hline Flight Mach Number & 0.78 & 0.25 & {$[-]$} \\
\hline
\end{tabular}

Table 2: Parameter variation of the performance model. Values of design point unless explicitly stated otherwise

\begin{tabular}{|c|c|c|c|c|c|}
\hline Name & Range & Unit & Name & Range & Unit \\
\hline Overall pressure ratio (OPR) & $38.0-39.0$ & {$[-]$} & \multirow{2}{*}{$\begin{array}{l}\text { Design outlet angle relative to the axis } \\
\text { of the inlet guide vane (IGV) }\end{array}$} & & \\
\hline Turbine inlet temperature $T_{4}$ & $1525.0-1565.0$ & {$[\mathrm{~K}]$} & & $16-21$ & [०] \\
\hline \multirow{2}{*}{$\begin{array}{l}\text { Pressure split of booster } \\
\text { and high pressure compressors }\end{array}$} & & & Inlet Mach number & $0.45-0.52$ & {$[-]$} \\
\hline & $0.81-0.84$ & {$[-]$} & Variation for the loading factor of each stage & $-0.1-0.1$ & {$[-]$} \\
\hline Bypass ratio (BPR) of the fan & $12.0-13.0$ & {$[-]$} & Reference incidence of all rotors and stators & $-2.0-2.0$ & [०] \\
\hline Area-averaged circ. flow & & & Hub-to-tip ratios at inlet & $0.57-0.6$ & {$[-]$} \\
\hline \multirow[t]{3}{*}{ speed $\left(\bar{u}_{c, I n}\right)$ at HPC inlet at MTO } & $372-378$ & {$[\mathrm{~m} / \mathrm{s}]$} & and outlet & $0.92-0.94$ & {$[-]$} \\
\hline & & & Scalers for the VGV-Schedules & & \\
\hline & & & of the first four stators & $0.8-1.2$ & {$[-]$} \\
\hline
\end{tabular}

Table 3: Parameter variation of the compressor aerodynamic design process (Meanline process)

Particularly interesting in this study is a consideration of the TSFC, as an important value of thermodynamics, and the SM as a quantity that depends on both disciplines.

The following results are based on an analysis with 20 trajectories, unless otherwise stated. All results are normalized to the highest value of $\mu^{*}$ and $\sigma$. The chosen number of trajectories is a compromise between the reliability of the meaningfulness of the characteristic values and the numerical effort of the simulations. It was determined by considering the statements of the Morris screening in relation to the number of trajectories.

Fig. 4 shows this study for an exemplary output parameter. It can be seen that the conclusions regarding the classification of the individual parameters do not change significantly from the use of 15 trajectories and that the use of 20 trajectories as a safe estimate should allow meaningful conclusions about the significance of the individual input parameters. 


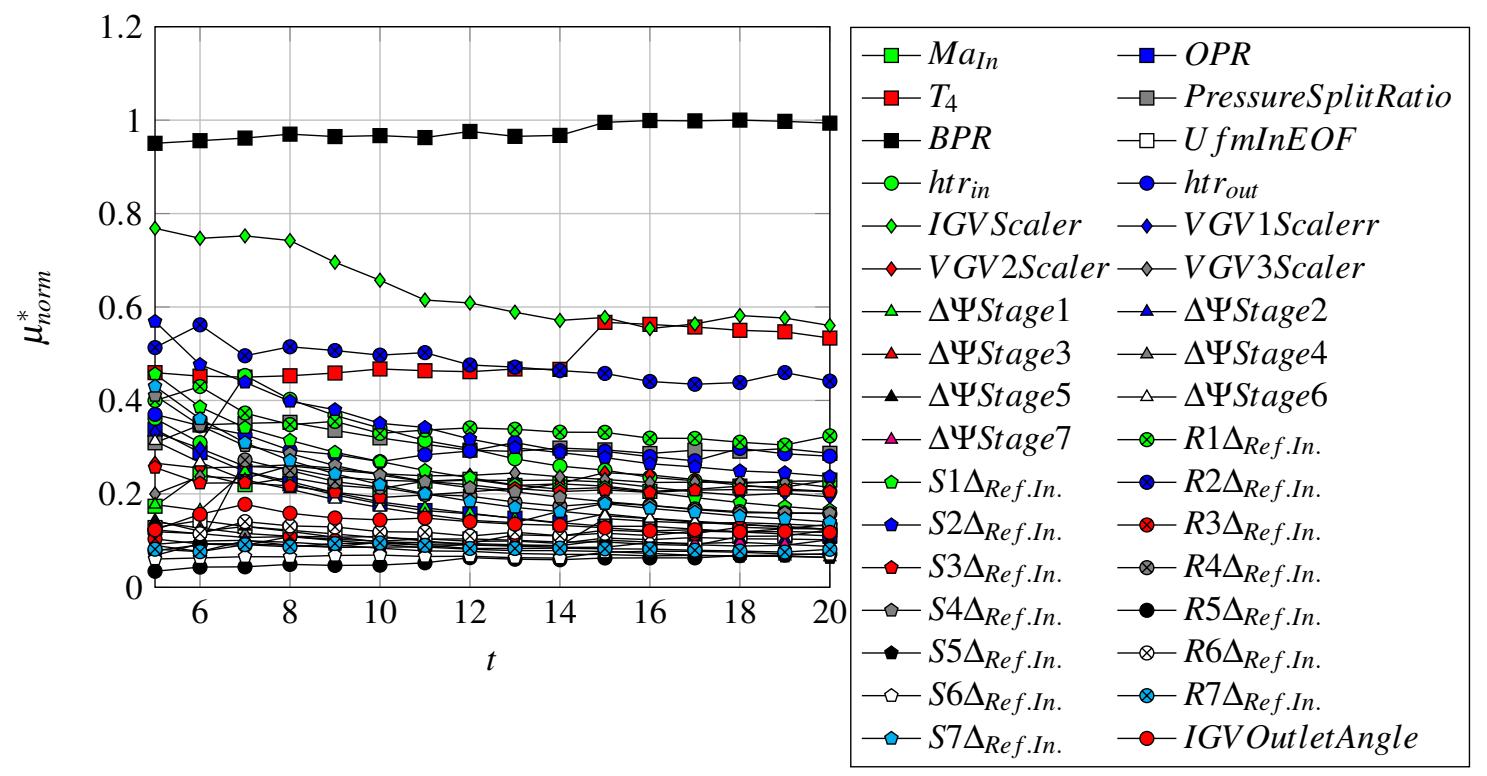

Figure 4: Morris screening evaluation for the output parameter TSFC at MTO over the number of evaluated trajectories

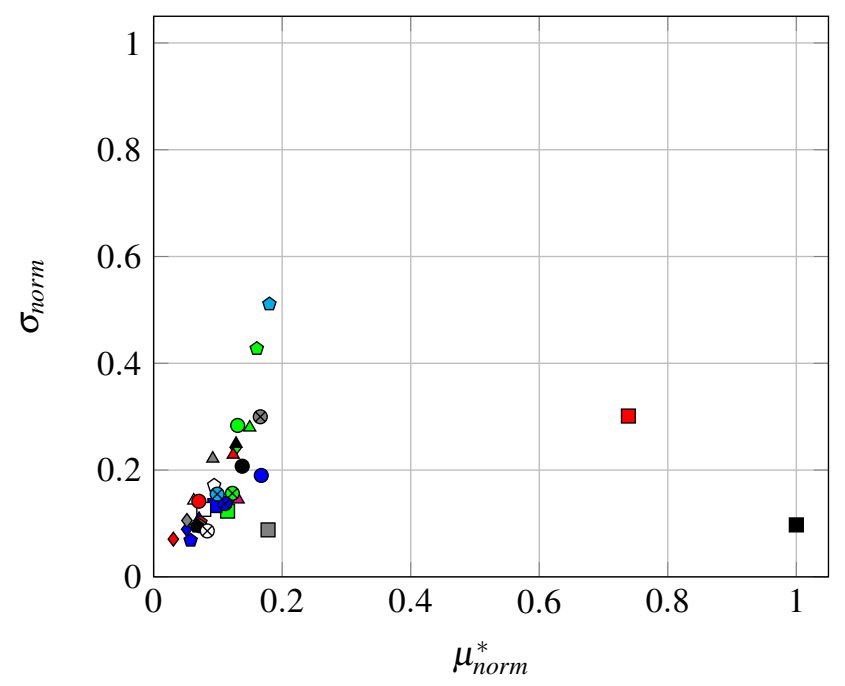

Figure 5: Morris screening evaluation for the output parameter TSFC (Design)

Results of Morris screening for TSFC shown in Fig. 5 for the design point and in Fig. 6 for the MTO as an exemplary off design operating point.

For the design point it is obvious that some of the thermodynamic input parameters of the study (squared symbols in the figure) are the most important parameters. Especially the variation of the BPR and turbine inlet temperature $\left(T_{4}\right)$ are influential. For the presented analysis for both parameters the absolute values of $\mu^{*}$ and $\mu$ are identical so the influence is monotone. For a rising $T_{4}$ within the considered limits the TSFC is increasing and for growing $B P R$ decreasing. The influence of T4 in this case can be explained by the counteracting effect of increasing internal efficiency and decreasing propulsion efficiency in the investigated design space.

Other parameters show a less clear or unambiguous influence. These are the pressure split ratio between the booster and the HPC and the hub-to-tip-ratio at the compressor outlet. Their influence is also purely monotonous. The Morris screening on the design efficiency of the HPC shows that these two parameters are the most influential there, and thus their influence on the TSFC at the design point can be explained.

On the other hand, a few parameters, which are mainly attributed to the off-design behaviour, have a clearly non-monotonous effect on the TSFC at the design point. Various deviations from the reference incidence and also the variations of the load distribution show compared to most other parameters relatively high values for $\sigma_{\text {norm. }}$. But a comparison of $\mu$ and $\mu^{*}$ shows that the first mentioned value is significantly smaller. The influence of these parameters on the TSFC at the design point can be explained by 


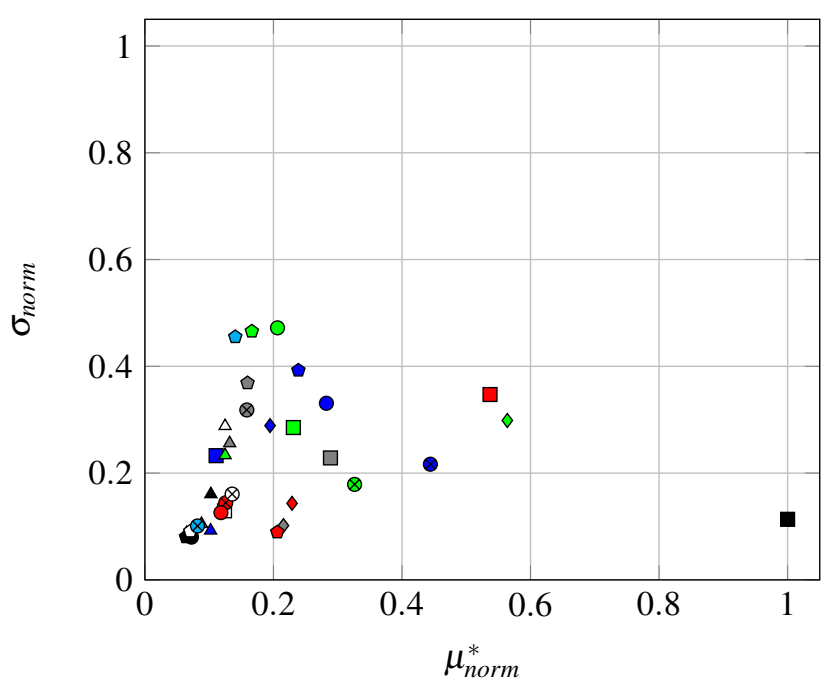

Figure 6: Morris screening evaluation for the output parameter TSFC OffDesign

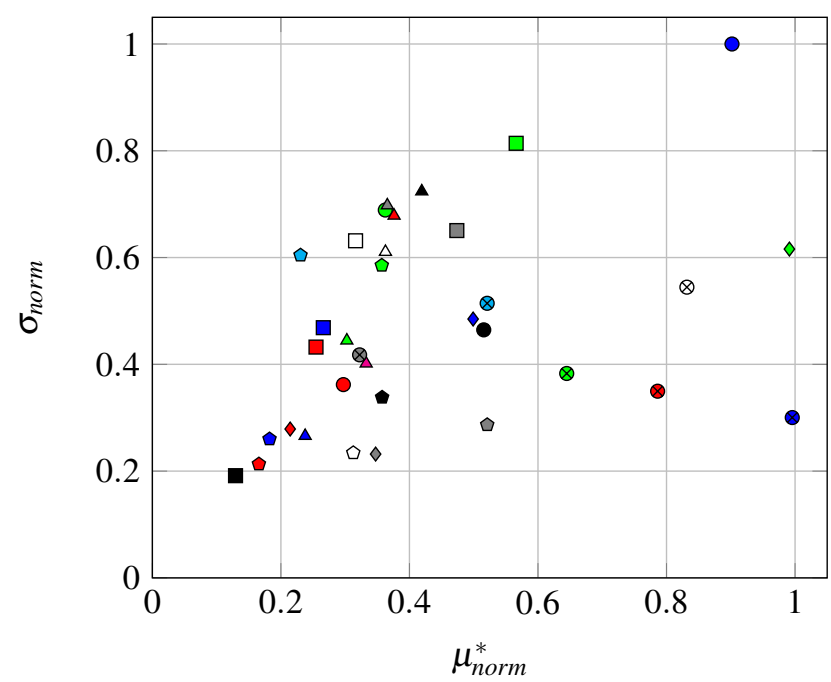

Figure 7: Morris screening evaluation for the output parameter SM OffDesign

feeding back the off-design behaviour via the map. The map of the HPC influences the results of the off-design operating points, which in turn establish boundary conditions for the design process, as explained above.

The TSFC for the MTO point as exemplary off design operating point is also as a feedback of the engine design most strongly influenced by the BPR and also clearly by the Mach number. In addition, the scaler for the schedule for adjusting the IGV also shows a significant influence. Furthermore, the variations in the reference incidence of the first two stages and the last stator as well as the hub-to-tip ratio also contribute to a lesser extent. The parameters for the variation of the loading coefficient and the reference incidences of the rear stages show a comparatively small influence.

Especially the parameters of the mean-line tool show non-linear and non-monotonic behaviour. This corresponds to the expected behaviour, that e.g. a deviation from the optimal blade flow by a variation of the reference incidence has a negative effect on the efficiency, but shows predominantly positive effects regarding the SM. The latter mentioned behaviour is visible in the analyses, too. The Morris screening results of the SM for the MTO operating point are shown in Fig. 7. Here the non-linear influences are shown by predominantly high values for $\sigma$. It can be seen that the reference incidences of the rotors have a much greater influence than those of the stators. Likewise, a clear influence of the IGV schedule scaler can be seen. Also the scaler for the schedule of the first stator is of greater importance, while the other schedule scalers have less influence.

Another variable examined is the number of stages which results for the compressor. It can be seen that a growing hub-to-tip ratio at the compressor outlet in particular has a decreasing effect on the number of stages. This is due to the target pressure ratio which can be better achieved at higher radii. Even though the influence is less pronounced, the inlet Mach number, the pressure split ratio and the hub-to-tip at the inlet are also of importance. However, these parameters show a monotonically increasing influence on the number of stages.

Overall, the analysis shows that the variation of the BPR and $T_{4}$ in particular have a significant influence on the overall system. Regarding the mean-line tool, the reference incidences of the front rotors and the scaler for the schedule of the IGV are of particular importance. If this study would serve as a preparation for further steps, e.g. an optimization, the parameter space could be sensibly reduced by the gained insight into the model. For example the parameters for the variation of the rear stages can be kept constant. As an exception, the analysis shows quite explicitly the influence of the reference incidence of the last stator. It remains to be said that only the influence in the defined interval ranges was examined, and a larger range for e.g. the OPR could also show a clearer impact.

\section{CONCLUSION}

In this work, a method for the full coupling of thermodynamic engine design and aerodynamic 1-D mean-line design of the high-pressure compressor was presented. The coupling by means of a compressor map allows a fast feedback of the compressor behaviour for different off-design operating points and thus consideration of their influence on the overall performance design. The method was used to demonstrate Morris screening. With this method, the influence of individual input parameters on result variables of a model can be estimated and compared.

The application example of the performance design of a two-spool unmixed turbofan engine coupled with the aerodynamic design of the HPC had shown that mainly the variation of parameters of the thermodynamic design influence results of the overall system e.g. the TSFC. However, the coupling with aerodynamics shows that also classical compressor design parameters, such like the hub-to-tip-ratio at the outlet, or a variation of the schedule for adjusting the IGV have a relatively large influence. 
In summary it can be said that Morris screening can help to classify the importance of the parameters within a large multidisciplinary parameter space. With the support of the determined characteristic values, meaningful decisions can be made, e.g. to reduce the parameter space, or insights can be gained into the monotony or linearity of influencing factors.

\section{REFERENCES}

Antinori, G., Muller, Y., Duddeck, F. and Fischersworring-Bunk, A. (2013), Statistical methods for a stochastic analysis of the secondary air system of a jet engine low pressure turbine, in 'ASME Turbo Expo 2013: Turbine Technical Conference and Exposition', American Society of Mechanical Engineers Digital Collection.

Arsenyev, I., Duddeck, F. and Fischersworring-Bunk, A. (2014), Global sensitivity analysis for multidisciplinary studies of vane clusters, in 'Vulnerability, Uncertainty, and Risk: Quantification, Mitigation, and Management', pp. 1554-1563.

Banjac, M., Petrovic, M. V. and Wiedermann, A. (2015), 'Secondary flows, endwall effects, and stall detection in axial compressor design', Journal of Turbomachinery 137(5).

Becker, R., Wolters, F., Otten, T. and Nauroz, M. (2011), 'Development of a Gas Turbine Performance Code and its Application to Preliminary Design', Deutscher Luft- und Raumfahrtkongress DLRK . Paper No. DLRK2011-241485.

Campolongo, F., Cariboni, J., Saltelli, A. and Schoutens, W. (2005), Enhancing the morris method, in 'Sensitivity Analysis of Model Output. Proceedings of the 4th International Conference on Sensitivity Analysis of Model Output (SAMO 2004)', pp. 369-379.

Chiba, K., Jeong, S., Obayashi, S. and Morino, H. (2005), Data mining for multidisciplinary design space of regional-jet wing, in '2005 IEEE Congress on Evolutionary Computation', Vol. 3, IEEE, pp. 2333-2340.

Eftari, M., Javaniyan, J. H., Shahhoseini, M. R., Ghadak, F. and Rad, M. (2011), 'Performance prediction modeling of axial-flow compressor by flow equations', Journal of Mechanical Research and Application (JMRA) .

Grieb, H. (2004), Projektierung von Turboflugtriebwerken, Birkhäuser Verlag, Basel, Switzerland. ISBN 978-3-0348-7938-5.

Iooss, B. and Lemaître, P. (2015), A review on global sensitivity analysis methods, in 'Uncertainty management in simulationoptimization of complex systems', Springer, pp. 101-122.

Khurana, M. and Winarto, H. (2010), 'Development and validation of an efficient direct numerical optimisation approach for aerofoil shape design', The Aeronautical Journal 114(1160), 611-628.

Kim, S., Kim, D., Son, C., Kim, K., Kim, M. and Min, S. (2015), 'A full engine cycle analysis of a turbofan engine for optimum scheduling of variable guide vanes', Aerospace Science and Technology 47, 21-30.

Lieblein, S., Schwenk, F. C. and Broderick, R. L. (1953), Diffusion factor for estimating losses and limiting blade loadings in axial-flow-compressor blade elements, Technical report, National Advisory Committee for Aeronautics, Lewis Flight Propulsion Laboratory, Cleveland, Ohio.

Morris, M. D. (1991), 'Factorial sampling plans for preliminary computational experiments', Technometrics 33(2), 161-174.

Nembrini, J., Samberger, S. and Labelle, G. (2014), 'Parametric scripting for early design performance simulation', Energy and Buildings 68, 786-798.

Østergård, T., Jensen, R. L. and Maagaard, S. E. (2017), 'Early building design: Informed decision-making by exploring multidimensional design space using sensitivity analysis', Energy and Buildings 142, 8-22.

Reitenbach, S., Becker, R., Hollmann, C., Wolters, F., Vieweg, M., Schmeink, J., Otten, T. and Siggel, M. (2020), 'Collaborative Aircraft Engine Preliminary Design using a Virtual Engine Platform, Part A: Architecture and Methodology', AIAA SciTech Forum .

Reitenbach, S., Schnös, M., Becker, R.-G. and Otten, T. (2015), Optimization of Compressor Variable Geometry Settings Using Multi-fidelity Simulation, in 'Proceedings of ASME Turbo Expo 2015: Turbine Technical Conference and Exposition'. ASME Paper No. GT2015-42832.

Saltelli, A., Ratto, M., Andres, T., Campolongo, F., Cariboni, J., Gatelli, D., Saisana, M. and Tarantola, S. (2008), Global sensitivity analysis: the primer, John Wiley \& Sons.

Schnoes, M. and Nicke, E. (2015), Automated Calibration of Compressor Loss and Deviation Correlations, in 'Proceedings of ASME Turbo Expo 2015: Turbine Technical Conference and Exposition'. ASME Paper No. GT2015-42644.

Templalexis, I., Pilidis, P., Pachidis, V. and Kotsiopoulos, P. (2011), 'Development of a two-dimensional streamline curvature code', Journal of turbomachinery 133(1). 